\title{
ORIGINAL ARTICLE \\ Late-life obesity is associated with smaller global and regional gray matter volumes: a voxel-based morphometric study
}

\author{
SJ Brooks ${ }^{1,6}$, C Benedict ${ }^{1,6}$, J Burgos ${ }^{1}$, MJ Kempton ${ }^{2}$, J Kullberg ${ }^{3}$, R Nordenskjöld ${ }^{3}$, L Kilander ${ }^{3}$, R Nylander ${ }^{4}$, E-M Larsson ${ }^{4}$, L Johansson ${ }^{4}$, \\ $\mathrm{H}$ Ahlström ${ }^{4}$, L Lind ${ }^{5}$ and HB Schiöth ${ }^{1}$
}

OBJECTIVE: Obesity adversely affects frontal lobe brain structure and function. Here we sought to show that people who are obese versus those who are of normal weight over a 5-year period have differential global and regional brain volumes. DESIGN: Using voxel-based morphometry, contrasts were done between those who were recorded as being either obese or of normal weight over two time points in the 5 years prior to the brain scan. In a post-hoc preliminary analysis, we compared scores for obese and normal weight people who completed the trail-making task.

SUBJECTS: A total of 292 subjects were examined following exclusions (for example, owing to dementia, stroke and cortical infarcts) from the Prospective Investigation of the Vasculature in Uppsala Seniors cohort with a body mass index of normal weight $\left(<25 \mathrm{~kg} \mathrm{~m}^{-2}\right)$ or obese $\left(\geqslant 30 \mathrm{~kg} \mathrm{~m}^{-2}\right)$.

RESULTS: People who were obese had significantly smaller total brain volumes and specifically, significantly reduced total gray matter (GM) volume (GMV) (with no difference in white matter or cerebrospinal fluid). Initial exploratory whole brain uncorrected analysis revealed that people who were obese had significantly smaller GMV in the bilateral supplementary motor area, bilateral dorsolateral prefrontal cortex (DLPFC), left inferior frontal gyrus and left postcentral gyrus. Secondary more stringent corrected analyses revealed a surviving cluster of GMV difference in the left DLPFC. Finally, post-hoc contrasts of scores on the trail-making task, which is linked to DLPFC function, revealed that obese people were significantly slower than those of normal weight.

CONCLUSION: These findings suggest that in comparison with normal weight, people who are obese have smaller GMV, particularly in the left DLPFC. Our results may provide evidence for a potential working memory mechanism for the cognitive suppression of appetite that may lower the risk of developing obesity in later life.

International Journal of Obesity (2013) 37, 230-236; doi:10.1038/ijo.2012.13; published online 31 January 2012

Keywords: VBM; DLPFC; trail-making task; working memory

\section{INTRODUCTION}

Epidemiological studies show that there is an association between obesity and cognitive impairment in otherwise cognitively healthy subjects. $^{1-3}$ Being obese as an adult worsens general cognitive performance ${ }^{4-6}$ and raises the risk of developing cognitive deficits later in life. ${ }^{7}$ Global brain volume reductions occur in people who are obese, ${ }^{8,9}$ and regionally, the frontal lobes are possibly the most susceptible to reductions that coincide with weight gain. ${ }^{10,11} \mathrm{~A}$ specific region of the frontal lobes, known as the dorsolateral prefrontal cortex (DLPFC) may be most vulnerable to volume deficits in those who are obese, as there is some evidence that cognitive functioning associated with the DLPFC is linked to the regulation of appetite. ${ }^{12,13}$ The DLPFC is involved in executive functioning, mainly working memory (WM) ${ }^{14,15} \mathrm{WM}$ is a process by which information about future goals is actively rehearsed for a brief period, while attending to immediate concerns. Artificial stimulation of the DLPFC with transcranial magnetic stimulation improves WM performance ${ }^{15}$ and lowers the experience of craving in those who are prone to binge eating. ${ }^{16}$ Increased gray matter volume (GMV) has been shown to be associated with increased regional brain activation, ${ }^{17}$ and so it is plausible that conversely, reduced regional brain volume is linked to deficient function However, it is also possible that greater volume in the DLPFC could also be observed in those who are obese, as they may need to apply greater cognitive restraint over their appetite, and employ more cognitive resources to achieve a similar level of cognitive performance as those who have healthy eating patterns.

In the most recent voxel-based morphometry (VBM) study of obesity effects on global and regional brain volumes, ${ }^{11}$ young obese females had lower GMV and WMV than lean and overweight counterparts. Furthermore, body mass index (BMI) correlated with increased WM volumes in temporal, visual and mesolimbic regions and decreased WM volumes in frontal regions. We progress these most recent VBM findings by examining the effects of late-life obesity on brain volume and cognition in a large cohort of elderly men and women. We hypothesized that late-life obesity, relative to normal weight is linked to global GM reduction. Additionally, as the DLPFC is linked to restraint of appetite, and reduced regional GMV has been recently observed in the obese, particularly in the frontal lobes, we also hypothesize regional reductions, particularly in the DLPFC.

${ }^{1}$ Department of Neuroscience, University of Uppsala, Uppsala, Sweden; ${ }^{2}$ Department of Neuroimaging, King's College, London, Decrespigny Park, London, UK; ${ }^{3}$ Department of Public Health and Caring Sciences/Geriatrics, University of Uppsala, Uppsala, Sweden; ${ }^{4}$ Department of Radiology, University of Uppsala, Uppsala, Sweden and ${ }^{5}$ Department of Medical Sciences, University of Uppsala, Uppsala, Sweden. Correspondence: Dr SJ Brooks, Department of Neuroscience, University of Uppsala, Box 593, Husargatan 3, 75124 , Uppsala, Sweden.

E-mail: drsamanthabrooks@gmail.com

${ }^{6}$ These authors contributed equally to this work.

Received 19 October 2011; revised 29 November 2011; accepted 21 December 2011; published online 31 January 2012 


\section{SUBJECTS AND METHODS}

\section{Participants}

Initially we screened 772 healthy elderly Swedish men and women who had a stable weight status over 5 years, as defined by their BMI $\left(\mathrm{kg} \mathrm{m}^{-2}\right)$ of obese $(\geqslant 30)$ or normal weight $(<25)$ at age 70 and 75 years were recruited from an epidemiological Swedish study named the Prospective Investigation of the Vasculature in Uppsala Seniors. ${ }^{18}$ Of these, as described below, a proportion also agreed to return for a brain scan using MRI at the age of 75 . The study was approved by the local Ethics Committee in Uppsala.

To ensure a cognitively healthy population, those with a history of dementia or stroke, those with pathological lesions on MRI (including arachnoidal cyst, bilateral hygromas, meningiomas and schwannoma) and those who scored $\leqslant 21$ on the mini-mental state examination ${ }^{19}$ were excluded to control for moderate cognitive impairment. Additionally, to compare those who were consistently obese with those who were consistently normal weight, we excluded those who had a weight diagnosis of overweight (that is, $\mathrm{BMI} \geqslant 25 \mathrm{~kg} \mathrm{~m}^{-2}$ or $<30 \mathrm{~kg} \mathrm{~m}^{-2}$ ), or who fluctuated between BMI classes during the 5 -year period of the study. Furthermore, we examined each individual's MRI scan, and we excluded one that was affected by movement artefacts. This meant that there were 292 cognitively healthy elderly Swedish men $(n=117)$ and women $(n=175)$ at age 75 years, and of these, 53\% $(n=156$, men $n=70$, women, $n=86$ ) had a MRI brain scan at age 75 years (Table 1 ).

\section{MRI acquisition}

Global and regional measures of brain volume were acquired with MRI and processed using VBM, a technique that uses statistical parametric mapping (SPM) to determine local concentrations of GM densities on a voxel-byvoxel basis. ${ }^{20} \mathrm{~A}$ high resolution three-dimensional $\mathrm{T} 1$-weighted volumetric turbo field echo scan was acquired using a Philips 1.5 Tesla scanner (Gyroscan NT, Philips Medical Systems, Best, The Netherlands). The threedimensional gradient echo sequence was used with scan parameters repetition time $8.6 \mathrm{~ms}$, echo time $4.0 \mathrm{~ms}$ and flip angle 8 degrees. Sagittal slices with a field of view of $240 \mathrm{~mm}$, a slice thickness of $1.2 \mathrm{~mm}$ and an in-slice resolution of $0.94 \times 0.94 \mathrm{~mm}$ were reconstructed.

\section{MRI processing}

Morphological changes in GM were calculated by segmenting GM from white matter and cerebrospinal fluid using the unified segmentation approach. ${ }^{21}$ Following this segmentation procedure, probability maps of

Table 1. Demographic data

\begin{tabular}{lcc}
\hline & $\begin{array}{c}B M I<25 \mathrm{~kg} \mathrm{~m}^{-2} \\
(\mathrm{n}=97)\end{array}$ & $\begin{array}{c}B M I \geqslant 30 \mathrm{~kg} \mathrm{~m}^{-2} \\
(\mathrm{n}=59)\end{array}$ \\
\hline Male/female (\%) & $46 / 54$ & $42 / 58$ \\
Type 2 diabetes $(\mathrm{n} / \%)$ & $8(8)$ & $18(31)^{* *}$ \\
University education $(\mathrm{n}, \%)$ & $29(30)$ & $13(22)^{* *}$ \\
BMl at 70 years $\left(\mathrm{kg} \mathrm{m}^{-2}\right)$ & $22.5 \pm 0.2$ & $33.0 \pm 0.3^{* *}$ \\
BMl at 75 years $\left(\mathrm{kg} \mathrm{m}^{-2}\right)$ & $22.1 \pm 0.2$ & $33.7 \pm 0.4^{* *}$ \\
TBV (ml) & $1022 \pm 10$ & $1007 \pm 15^{*}$ \\
GM (ml) & $572 \pm 6$ & $563 \pm 10^{*}$ \\
TMT-A (s) & $59 \pm 3$ & $60 \pm 3$ \\
TMT-B (s) & $169 \pm 12$ & $161 \pm 14$ \\
TMT-B-TMT-A (s) & $110 \pm 11$ & $100 \pm 12$ \\
MMSE & $28.6 \pm 0.1$ & $28.8 \pm 0.2$ \\
\hline ABMSE
\end{tabular}

Abbreviations: $\mathrm{BMI}$, body mass index; $\mathrm{GM}$, gray matter; MMSE, mini-mental state examination; TBV, total brain volume; TMT-A, trail-making test $A$ (normal completion time $=\sim$ between 30 and $90 \mathrm{~s}$ ); TMT-B, trail-making test $\mathrm{B}$ (normal completion time $=\sim$ between 75 and $180 \mathrm{~s}$ ). Mean values ( \pm s.e.m.) are presented, if not otherwise stated. Normal-weight $\left(\mathrm{BMl}<25 \mathrm{~kg} \mathrm{~m}^{-2}\right)$ and obese $\left(\mathrm{BMl} \geqslant 30 \mathrm{~kg} \mathrm{~m}^{-2}\right)$ elderly men and women at both 70 and 75 years of age were included. ${ }^{*} P<0.05$. ${ }^{*} P<0.01$ for comparisons between obese and lean.
GM were 'modulated' to account for the effect of spatial normalization by multiplying the probability value of each voxel by its relative volume in native space before and after warping. GM images based on probability maps at each voxel were normalized into Montreal Neurological Institute standard space for further contrast analysis between the obese and lean groups. Following contrast analysis, Montreal Neurological Institute coordinates were converted from Montreal Neurological Institute to Talairach space using the mni2tal script (http://www.imaging.mrc-cbu.cam. ac.uk/imaging/MniTalairach). Modulated images were smoothed with an 8-mm full width half maximum (FWHM) Gaussian kernel, in line with other recent VBM studies. ${ }^{22}$ This smoothing kernel was applied prior to statistical analysis to reduce signal noise and to correct for image variability.

\section{VBM contrasts}

Our VBM contrasts were between those who were obese versus those who were normal weight, between two time points over a 5-year period. The first level analysis was an uncorrected whole brain contrast to examine overall, general differential brain volume between the groups. Then we conducted second level analysis by applying more stringent corrections using a family-wise error, small volume correction and a threshold of 100 voxels. We corrected all VBM contrasts for multiple comparisons, gender, brain matter volume (gray and white matter), education status (nonuniversity versus university) and diabetes status. VBM analyses were carried out using SPM8-VBM toolbox (VBM 8.1 version 1.20; http:// www.fil.ion.ucl.ac.uk/spm/). ${ }^{20}$ Volume data in $\mathrm{ml}$ was extracted for total GM, total white matter and regional volumetric data in the left DLPFC, using the SPM region of interest extraction tool MarsBaR (MARSeille Boite Á Région d'Intérét), version 0.42 (http://www.marsbar.sourceforge.net).

\section{Statistical analyses}

Data was analyzed using multifactorial analyses of covariance in IBM SPSS Statistics version 19 for Windows (http://www-01.ibm.com/software/ analytics/spss). Where appropriate, post-hoc tests were conducted using univariate analyses of covariance, $\chi^{2}$ - and $t$-tests. Gender, type 2 diabetes and level of educational attainment (university level versus primary and secondary level education) were used as covariates of no interest.

\section{Post-hoc cognitive experiment}

In our VBM results (described below), we found a highly significant (uncorrected and corrected) GM reduction in the left DLPFC, a region associated with WM performance. Thus, as our cohort also took part in the trail-making test (TMT), a cognitive task that measures WM performance (and also spatial navigation and attention), which is associated with DLPFC function, ${ }^{12,13}$ we decided to examine post-hoc as a first exploratory analysis, the cognitive performance on the TMT in the 292 cognitively healthy elderly Swedish men $(n=117)$ and women $(n=175)$ who did the task. Additionally, as a second exploratory analysis, we examined the $53 \%$ $(n=156)$ of people who had a brain scan $(-21$ people who did not do both the brain scan and cognitive task) to see whether TMT performance correlated with brain volume. To do this, we created a post-hoc anatomical mask of the left middle and superior temporal gyrus in the left hemisphere as well as a distinct left-sided dorsolateral and medial frontal region, in line with a recent functional MRI study showing that these regions are most activated during the TMT. ${ }^{23}$ We deemed this mask particularly relevant, given our initial uncorrected findings. The mask was created using the SPM toolbox function PickAtlas (http://www.fmri.wfubmc.edu/software/ PickAtlas) and by selecting Brodmann areas 9, 21 and 46.

TMT. The TMT is a combined test of executive function (for example, mental speed, divided attention and cognitive flexibility) with varying difficulty (that is, TMT version A (TMT-A) and TMT version B (TMT-B)), which is associated with frontal lobe function. ${ }^{24}$ During the TMT-A, participants are required to join the dots representing 25 targets of ascending numbers $(1-2-3-4-5 \ldots)$ on a sheet of paper. For the TMT-B, participants respond in the same manner, but instead alternate between a set of numbers $(1-13)$ and a set of letters $(A-L)$, again in ascending order $(1-A-2-B-$ $3-C-4-D-5 \ldots)$. In essence, the TMT-B task adds a set-shifting component 
whereby participants must continually shift between two different strategy sets. The participants must start their trial on the circle marked 'Begin', and continue until they reach the endpoint, a circle marked 'End'. The goal is to complete the task as quickly as possible, and the outcome measurement is time in seconds. It is recommended practice to subtract the TMT-A score from the TMT-B score, to create a difference score, in order to correct for individual variability in overall completion speed. ${ }^{24}$

When examining the TMT, we used a one-tailed test for significance as there was a priori evidence that the obese group would be expected to

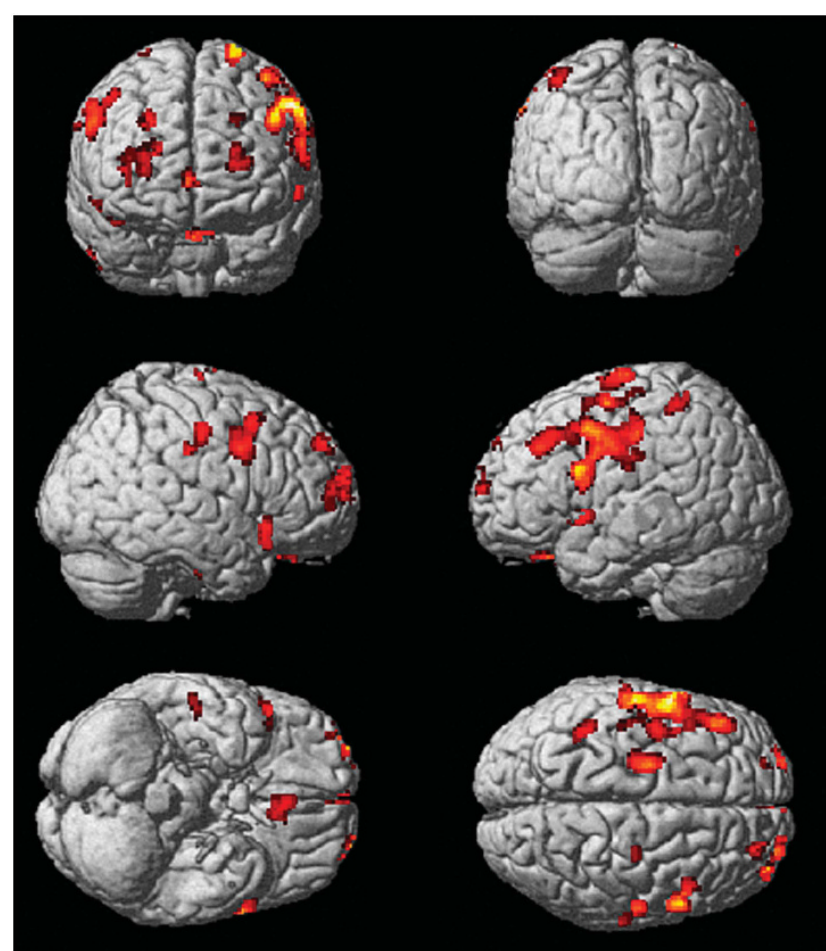

Figure 1. The influence of obesity on bilateral DLPFC, bilateral SMA, left IFG and left postcentral gyrus (PCG) (uncorrected). Rendered image of VBM illustrates significant GMV reductions in frontotemporal regions in obese elderly men and women $(n=59)$, as compared with normal-weight subjects $(n=97)$. These analyses were uncorrected and only clusters significant at $P<0.05$ were reported. perform worse than the normal-weight group. ${ }^{4}$ Furthermore, in a step to correct for false positives, we excluded those individuals whose TMT scores were outliers, greater than two s.d. from the population mean $(n=15)$. We chose a more stringent two, rather than three s.d. as a cut off for cognitive performance, because we felt that other factors might influence our cognitive score in this elderly cohort, for example, slow movement in the hands, reduced vision (although all participants reported being able to see the targets). Thus, by using a more stringent cut off, we can account for some of the variance that might be caused by factors other than variance in cognitive ability. The level of significance was set to $P<0.05$.

\section{RESULTS}

\section{Brain volumes}

We found that people who are obese have an approximately 1 $47 \%$ significant reduction in total brain volume, $P<0.05$, and an approximately $1.57 \%$ significant reduction in total GM volume, $P<0.05$, compared with those who are of normal weight. Additionally, gender was found to be a nonsignificant covariant for brain volumes in our elderly Swedish cohort (Table 1).

\section{Uncorrected whole brain analyses}

Initial uncorrected whole brain analysis revealed 11 peak voxels indicating potential brain volume differences in: the left supplementary motor area (SMA, Brodmann area 6 cluster size: $1696 \mathrm{~mm}^{3}$, Talairach coordinates $=-56,0,42, P<0.0001$ ), left inferior frontal gyrus (IFG, Brodmann area 44, cluster size: $1696 \mathrm{~mm}^{3}$, Talairach coordinates $=-58,8,20, P=<0.0001$ ), left DLPFC (Brodmann area 9, cluster size: $1696 \mathrm{~mm}^{3}$, Talairach coordinates $=-44,32,34, P<0.0001$ ), right DLPFC (Brodmann area 9, cluster size: $798 \mathrm{~mm}^{3}$, Talairach coordinates $=28,54,38$, $P<0.001$ ), right medial prefrontal cortex (Brodmann area 10 cluster size: $798 \mathrm{~mm}^{3}$, Talairach coordinates $=32,64,20$ and 24, 68, 22, $P<0.001$ ), right DLPFC (Brodmann area 9, cluster size: $352 \mathrm{~mm}^{3}$, Talairach coordinates $=56,6,34$ ), right SMA (Brodmann area 6, cluster size: $352 \mathrm{~mm}^{3}$, Talairach coordinates $=46,12,20, P<0.01$ ), left SMA (Brodmann area 6, cluster size: $243 \mathrm{~mm}^{3}$, Talairach coordinates $=-42,-4,60$, $P<0.05$ ), left SMA (Brodmann area 6, cluster size: $243 \mathrm{~mm}^{3},-40$, $6,60, P<0.05$ ) and the left postcentral gyrus (Brodmann area 3 , cluster size $243 \mathrm{~mm}^{3}$, Talairach coordinates $=-46,-18,66$, $P<0.05$ ) (Figure 1, Table 2).

Table 2. Significant differences in gray matter volume between obese and lean elderly men and women, exploratory uncorrected whole brain analysis

\begin{tabular}{|c|c|c|c|c|c|c|c|c|}
\hline \multirow[t]{2}{*}{ Contrast } & \multirow[t]{2}{*}{ Brain region } & \multirow[t]{2}{*}{ Brodmann area } & \multirow[t]{2}{*}{ Cluster size $\left(\mathrm{mm}^{3}\right)$} & \multicolumn{3}{|c|}{ Peak voxel Talairach coordinates } & \multirow[t]{2}{*}{$T$} & \multirow[t]{2}{*}{$\mathrm{P}_{\text {uncorrected }}$} \\
\hline & & & & $x$ & $y$ & $z$ & & \\
\hline \multicolumn{9}{|l|}{ Lean > obese } \\
\hline & Left supplementary motor area & 6 & 1696 & -56 & 0 & 42 & 4.58 & 0.000 \\
\hline & Left inferior frontal gyrus & 44 & & -58 & 8 & 20 & 4.48 & 0.000 \\
\hline & Left dorsolateral prefrontal cortex & 9 & & -44 & 32 & 34 & 4.26 & 0.000 \\
\hline & Right dorsolateral prefrontal cortex & 9 & 798 & 28 & 54 & 38 & 4.37 & 0.001 \\
\hline & Right medial prefrontal cortex & 10 & & 32 & 64 & 20 & 4.09 & 0.001 \\
\hline & Right medial prefrontal cortex & 10 & & 24 & 68 & 22 & 3.79 & 0.001 \\
\hline & Right dorsolateral prefrontal cortex & 9 & 352 & 56 & 6 & 34 & 4.34 & 0.017 \\
\hline & Right supplementary motor area & 6 & & 46 & 12 & 50 & 3.51 & 0.017 \\
\hline & Left supplementary motor area & 6 & 243 & -42 & -4 & 60 & 3.85 & 0.041 \\
\hline & Left supplementary motor area & 6 & & -40 & 6 & 60 & 3.05 & 0.041 \\
\hline & Left postcentral gyrus & 3 & & -46 & -18 & 66 & 2.87 & 0.041 \\
\hline Obese $>$ lean & - & & - & - & - & - & - & - \\
\hline
\end{tabular}




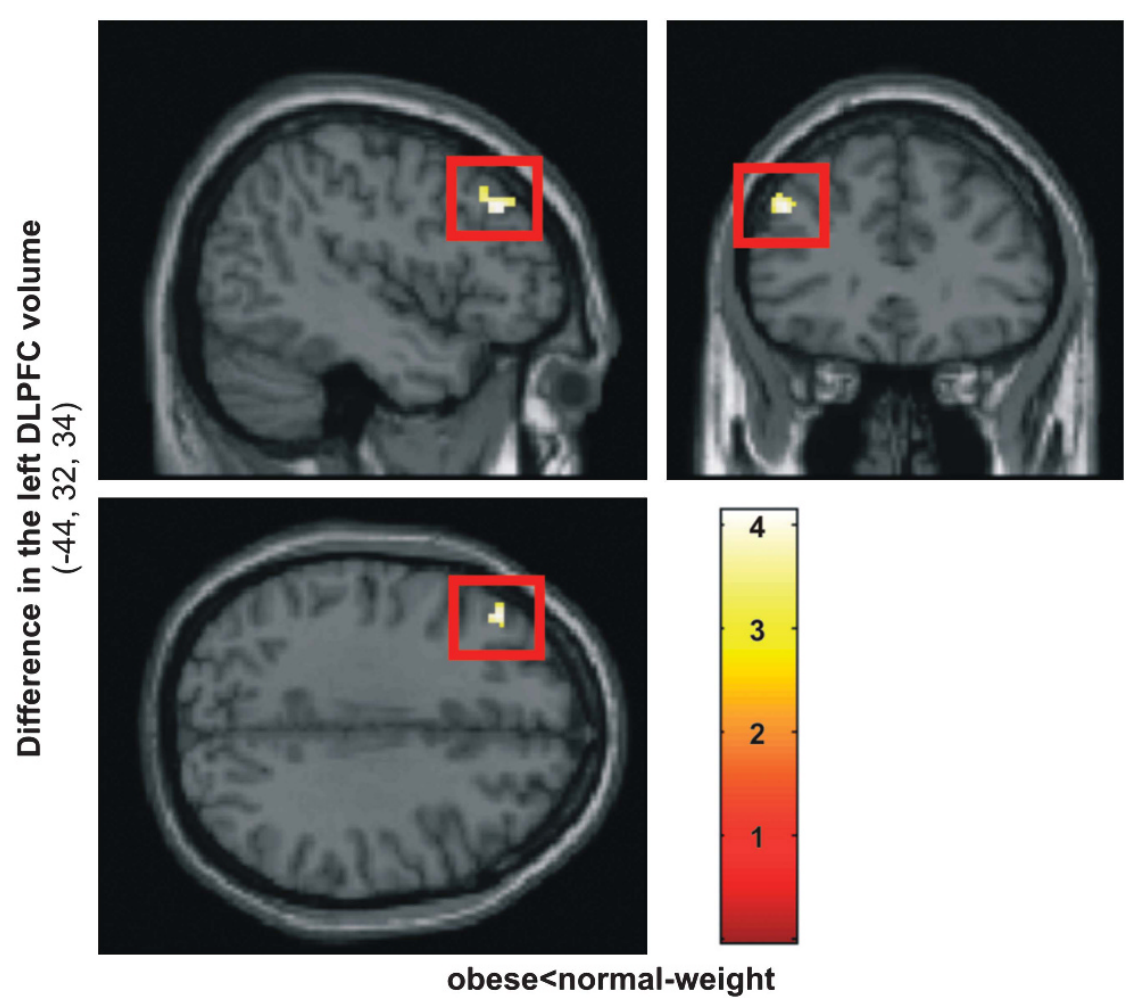

Figure 2. The influence of obesity on the dorsal prefrontal cortex GMV in elderly men and women (corrected). Brain slices of VBM illustrate significant GMV reductions in the left dorsolateral prefronal cortex (DLPFC) in obese elderly men and women ( $n=59$ ), as compared with normal-weight counterparts ( $n=97$; saggital (left), coronal (right) and axial (bottom) slices). Multiple voxel comparisons were corrected using the family-wise error (FWE) threshold of $P<0.05$, and a cluster threshold of 50 voxels was applied. Color bar indicates $T$ values.

Table 3. Significant differences in gray matter volume between obese and lean elderly men and women, FWE-corrected, $100 \mathrm{~mm}^{3}$ cluster threshold applied

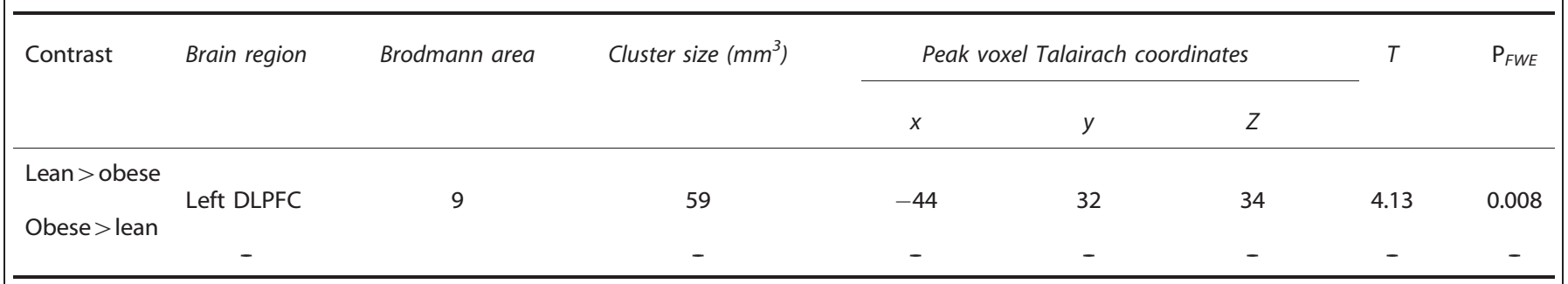

Abbreviations: DLPFC, dorsolateral prefrontal cortex; FEW, family-wise error. Multiple voxel comparisons using voxel-based morphometry, and respective

$P$-values are FWE-corrected. Education served as covariate of no interest.

Corrected region of interest analyses

On the basis of our findings from the uncorrected whole brain analyses, we created a post-hoc mask that included fronto-temporal regions as regions of interest. We then re-ran the analysis, corrected for family-wise error and applied a cluster threshold of 100 voxels. This produced a brain map of regional GM reductions in the obese compared with the normal-weight group in one major cluster comprising the left DLPFC, Brodmann area 9, cluster size: $59 \mathrm{~mm}^{3}$, Talairach coordinates $-44,32$, 34; peak level, $P<0.008$, family-wise error corrected for mask volume; Figure 2 . No other brain regions were significantly different between the obese and normal-weight groups. This brain region was also one of the strongest clusters of differential brain volume between obese and normal weight in the initial uncorrected whole brain analysis (Table 3).

\section{TMT}

The obese group tended to be slower when completing the TMT-B task relative to the normal-weight group $(P<0.07$, one-tailed, corrected for gender, diabetes and education). However, the analyses of covariance revealed that neither gender nor diabetes significantly influenced the TMT-B task performance $(P>0.45$ and $P>0.23$, respectively), whereas education did $(P<0.006)$. Thus, we re-ran a second analyses of covariance model including only education as a covariate. This yielded a significant effect of BMI on the TMT-B performance in that the obese group were slower to join the dots by approximately $11 \%$ as compared with the normalweight group $(P<0.04$, one-tailed, corrected for education, see Figure 3). However, in our correlation analysis between TMT-B performance and brain volume, we found no significant relationship between either global or regional GMV.

\section{DISCUSSION}

We found that being obese, as compared with being of normal weight late in life is associated with global GM reduction as well as regional GM reductions in the bilateral DLPFC, bilateral SMA, left 


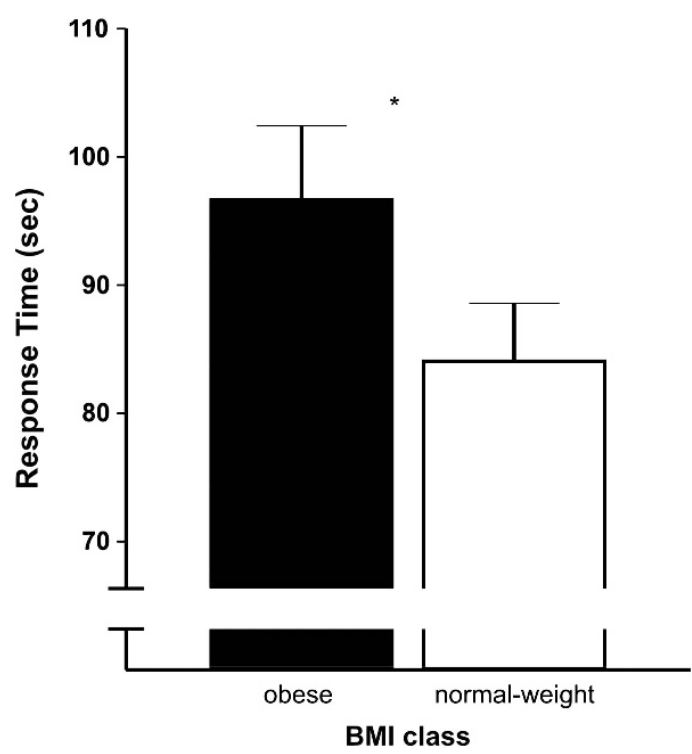

Figure 3. TMT performance in obese and normal-weight elderly men and women. Time taken to complete the trail-making $B$ test in seconds (corrected for the trail-making A test performance), in a total of 292 stable (at both age 70 and 75 years) obese $\left(\mathrm{BMI} \geqslant 30 \mathrm{~kg} \mathrm{~m}^{-2}, n=112\right)$ and normal-weight (BMI $<25 \mathrm{~kg} \mathrm{~m}^{-2}$ $n=180$ ) elderly Swedish men and women. ${ }^{*} P<0.04$, one tailed after correction for university education.

IFG and left postcentral gyrus. The reduced GMV in the left DLPFC was the most robust finding (in both global uncorrected and regional corrected analyses), and we also present some preliminary evidence that the obese group might have a WM deficit. This is particularly tantalizing, given that the left DLPFC is a brain region sub-serving WM function in humans. ${ }^{25}$ However, we were unable to correlate WM performance with brain volume, and this might be because of our smaller sample size in the scanned subpopulation, or other late-life factors, for example, arthritis or vasculature deficits leading to reduced nerve sensitivity in the hands, slowing performance on the task. Additionally, the lack of correlation between the trail making $B$ task and brain volume could indicate that cognitive function correlates better with brain function than brain structure, which is inherently static and subject to organic variability that might be independent of cognitive performance, but dependent on other lifestyle factors (for example, diet, exercise, hereditary factors and lifetime stressors). Nevertheless, our results may provide preliminary evidence for a potential mechanism as to why obese humans are unable to control their food intake ${ }^{12,13}$ and are more prone to develop cognitive impairment. ${ }^{7,26,27}$

Although a few other VBM studies have shown that increased weight is linked to global and regional GM reductions (for example, in the temporal gyrus, visual cortex, parahippocampal gyrus and dorsal striatum), ${ }^{8,9,11}$ which may have a genetic link, ${ }^{10}$ to our best knowledge only one study to date has examined the link between regional GMV volume reduction and how this might link to cognitive deficits in a population of obese people. ${ }^{22}$ However, this previous study differs to ours in that they only measure 95 females, whereas we have measured 292 males and females, $156(53 \%)$ of whom also had a brain scan. Additionally, the brain scans of our cohort were all done at age 75, whereas in the previous study there was a range of those who were between 52-92 years of age, which could potentially confound the results. Furthermore, we chose to do two levels of contrast analysis using only obese versus normal weight: one using a whole brain uncorrected analysis and a second using a more stringent correction with family-wise error, a voxel threshold and a small volume correction, whereas the previous study used a multivariate linear regression analysis, including normal weight, overweight and obese. A contrast analysis as opposed to a regression analysis might be able to highlight more prominent differences in brain volume. Additionally, and unlike the previous study, as many of our elderly cohort, particularly in the obese group were formally diagnosed with type 2 diabetes, we controlled for diabetes as well as gender, total brain volume and level of education, in line with other studies. ${ }^{8,9}$ In the previous study, it was found that obesity is linked to reductions of the left orbitofrontal cortex, right IFG, right precentral gyrus, parahippocampal, fusiform and lingual gyri, as well as reduced right cerebellar volume and WM deficits. We did not find reductions in these regions, although we did find other frontal lobe reductions, but this could be because of the methodological differences described above.

Other studies have found that BMI not only correlates with regional $\mathrm{GM}$ reductions in obese men in the bilateral temporal lobes, anterior cerebellum, parietal lobe, occipital lobe, frontal lobe and putamen ${ }^{8,9}$ but also correlates with increased volume in the inferior frontal gyri, posterior cerebellum and caudate. ${ }^{9}$ Others found that the superior and middle frontal gyri were most susceptible to BMI increases in a 1-year follow-up study. ${ }^{11}$ A major difference in our study was that instead of correlating GM with BMI we chose to examine those with high and low BMls over a stable 5-year period. This enabled us to replicate the finding that the frontal gyri, particularly the DLPFC, are mostly reduced in those who are obese. Methodologically, most other VBM studies in to obesity effects on the brain have examined younger cohorts, sometimes in only one gender. Here, we aimed to examine whether similar effects pertain to an older population of both males and females (corrected for gender differences). In a similar vein to the other studies, we found reductions in the frontal and parietal regions in those who were obese. But we also found reduced SMA, unlike in most other studies with younger cohorts. This might suggest that being obese later in life has a greater detrimental effect on mobility than if one is obese at a younger age and could exacerbate common old-age illnesses.

We found global GM reduction in the obese group, which is in line with previous findings ${ }^{28}$ particularly in the frontotemporal region, an area that is susceptible to neurodegeneration and dementia in the elderly. ${ }^{29}$ Additionally, magnetic resonance spectroscopy studies have also revealed that a higher BMI correlates with lower concentrations of a spectroscopic marker of neuronal variability in frontal, parietal and temporal lobes. ${ }^{30,31}$ We also found GM reductions in the bilateral SMA, left IFG and left postcentral gyrus, all regions associated with motor functions. The cerebellum, which is associated with motor coordination, was also found to be reduced in the previous study. ${ }^{22}$ One could speculate that as being obese may discourage a person from being physically active, it might be that reduced motor function has a detrimental effect on corresponding brain regions, but one cannot be certain of directionality in correlation analysis. An increased $\mathrm{BMI}$ is also shown to correlate with decreases in the right IFG, ${ }^{22}$ and in those who have neurodegeneration in similar frontal and temporal regions, specific problems with overeating are observed. $^{32}$ Thus, in our otherwise cognitively healthy cohort of elderly men and women, regional GM reduction in frontotemporal regions may underlie, or be driven by problematic eating behavior, lack of physical exercise and subsequent weight gain.

Our most robust region-specific GM reduction in those who were obese was found in the left DLPFC. The function of the DLPFC has been linked to successful appetite regulation, ${ }^{12}$ and artificial stimulation of the left DLPFC reduces cue-induced craving for food. ${ }^{16}$ Also, the left DLPFC has been associated with reduced function in those who are obese. ${ }^{13}$ Furthermore, brain structure and function have been shown to be significantly correlated, ${ }^{17}$ so it is conversely plausible that degeneration of this region is associated with reduced function, unsuccessful appetite 
regulation and greater craving for food, which potentially leads to obesity. Reduced activation of the DLPFC is associated with an impaired ability to control the hyperconsumption of food, $^{33}$ especially in those who are obese. ${ }^{13}$ However, caution must be applied when commenting on the direction of this link, as being obese could also potentially lead to brain structure changes, particularly if the integrity of the vasculature is compromised. From a similar vein, obesity could also be associated with an increased volume in the DLPFC, as greater restraint is needed over appetite or greater recruitment of attention to complete cognitive tasks to the same level as a person who is less prone to impulsive responses. However, at least in an elderly cohort, obesity does seem to be associated with reduced brain volume in the DLPFC. Obesity and vasculature disorders such as hypertension, diabetes and cardiac dysfunction are often associated, elevating the risk of developing Alzheimer's disease in old age. ${ }^{34}$ Thus, it is plausible that suboptimal functioning in the frontal lobes are associated with genetic risk, unhealthy responses to environmental food cues and vasculature disorders that ultimately lead to the development of obesity and cognitive decline, particularly in old age.

Methodological differences must also be taken in to account when interpreting our findings in light of previous studies. For example, some studies use only a region of interest approach, for example, ${ }^{11}$ whereas we have conducted both global and regional analyses, which are not biased by a priori assumptions. However, we did find similar regions of reduction in obese, to those observed in regions of interest studies (frontal regions), and so it seems our findings are robust. Furthermore, we did not correlate brain volume with BMI, but contrasted those who were obese with those who were normal weight over a 5-year period between the ages of 70-75 years. Yokum and Stice ${ }^{11}$ recently found that a region of the frontal lobes, also involving the DLPFC, was most susceptible to reduction in a 1-year follow-up of increasing obesity. Thus, our contrast analyses showing that the left DLPFC is the most robust area of reduced volume in those who were obese over a 5-year period is corroborated by other analytical methods. Finally, there can be differences in statistical analysis of VBM data (for example, pre-processing, size of smoothing kernels) that may influence the results, and also, anatomical differences between contrast groups (for example, obese versus normal weight) may alter the image intensities reported that are independent of actual structural differences. Nevertheless, with these methodological differences in mind, our data seem to fit well with previous VBM studies of brain volume in obesity, in that frontal brain regions seem to be most susceptible to reduction.

\section{STRENGTHS AND LIMITATIONS}

There are strengths and limitations to this study that must be considered; first, it was conducted on a homogenous, communitybased population with a careful assessment of potential confounding variables. However, one major limitation is that it is difficult to extrapolate our results to other age groups, and because of the cross-sectional nature of our study, it is difficult to draw conclusions about any potential cause-effect relationships. Furthermore, we cannot avoid the possibility that obese elderly people may suffer from more diseases than those who were normal weight, and that this could contribute to poor performance on the WM task as well as structural differences. Additionally, we did not measure fluid intelligence (intelligence quotient) and so cannot exclude the possibility that intelligence may have contributed to some of the cognitive effects observed. However, all of our subjects were cognitively healthy, and so we were able to reveal a potential link between brain volume, BMI and cognitive function. Another strength of this study is that we conducted both uncorrected and corrected whole brain and regions of interest analyses, which demonstrated a robust difference in the left DLPFC, a brain region that is functionally linked to the TMT task. ${ }^{23}$

\section{CONCLUSION}

Our results suggest that reduced brain volume in the left DLPFC is the most robust deficit in those who are obese, and that a WM deficit might be driven by being obese, or contribute to unsuccessful weight regulation in the elderly.

\section{CONFLICT OF INTEREST}

The authors declare no conflict of interest.

\section{ACKNOWLEDGEMENTS}

The study was funded by Swedish Research Council (including K2010-64X-0826823-3), Brain Research Foundation, Novo Nordisk, Tore Nilsons foundation, Gunvor och Josef Anérs stiftelse, and Åhlens foundation, AstraZeneca (Sweden). The funding sources had no input in the design and conduct of this study, in the collection, analysis and interpretation of the data, or in the preparation, review or approval of the manuscript.

\section{AUTHOR CONTRIBUTIONS}

$\mathrm{LL}, \mathrm{HA}, \mathrm{LH}, \mathrm{EML}$ and $\mathrm{RN}$ conceived and designed the experiments. $\mathrm{L}, \mathrm{LL}, \mathrm{HA}$, $J K, R N, R N$ and EML performed the experiments. SJB, CB and JB analyzed the data. All authors were responsible in the writing of this paper or in intellectual contributions.

\section{REFERENCES}

1 Elias MF, Elias PK, Sullivan LM, Wolf PA, D'Agostino RB. Obesity, diabetes and cognitive deficit: the Framingham Heart Study. Neurobiol Aging 2005; 26 (Suppl 1): $11-16$.

2 Elias MF, Elias PK, Sullivan LM, Wolf PA, D'Agostino RB. Lower cognitive function in the presence of obesity and hypertension: the Framingham heart study. Int J Obes Relat Metab Disord 2003; 27: 260-268.

3 Cohen RA. Obesity-associated cognitive decline: excess weight affects more than the waistline. Neuroepidemiology 2010; 34: 230-231.

4 Gunstad J, Paul RH, Cohen RA, Tate DF, Spitznagel MB, Gordon E. Elevated body mass index is associated with executive dysfunction in otherwise healthy adults. Compr Psychiatry 2007; 48: 57-61.

5 Boeka AG, Lokken KL. Neuropsychological performance of a clinical sample of extremely obese individuals. Arch Clin Neuropsychol 2008; 23: 467-474.

6 Volkow ND, Wang GJ, Telang F, Fowler JS, Goldstein RZ, Alia-Klein N et al. Inverse association between BMI and prefrontal metabolic activity in healthy adults. Obesity (Silver Spring) 2009; 17: 60-65.

7 Kivipelto M, Ngandu T, Fratiglioni L, Viitanen M, Kareholt I, Winblad B et al. Obesity and vascular risk factors at midlife and the risk of dementia and Alzheimer disease. Arch Neurol 2005; 62: 1556-1560.

8 Pannacciulli N, Del Parigi A, Chen K, Le DS, Reiman EM, Tataranni PA. Brain abnormalities in human obesity: a voxel-based morphometric study. Neuroimage 2006; 31: 1419- 1425.

9 Taki Y, Kinomura S, Sato K, Inoue K, Goto R, Okada K et al. Relationship between body mass index and gray matter volume in 1,428 healthy individuals. Obesity (Silver Spring) 2008; 16: 119-124.

10 Ho AJ, Stein JL, Hua X, Lee S, Hibar DP, Leow AD et al. A commonly carried allele of the obesity-related FTO gene is associated with reduced brain volume in the healthy elderly. Proc Natl Acad Sci USA 2010; 107: 8404-8409.

11 Yokum S, $\mathrm{Ng} \mathrm{J}$, Stice E. Relation of regional gray and white matter volumes to current BMI and future increases in BMl: a prospective MRI study. Int J Obes (Lond) 2011; e-pub ahead of print 6 September 2011; doi: 10.1038/ijo.2011.175.

12 Hollmann M, Hellrung L, Pleger B, Schlogl H, Kabisch S, Stumvoll M et al. Neural correlates of the volitional regulation of the desire for food. Int J Obes (Lond) 2011; e-pub ahead of print 28 June 2011; doi: 10.1038/ijo.2011.125.

13 Le DS, Chen K, Pannacciulli N, Gluck M, Reiman EM, Krakoff J. Reanalysis of the obesity-related attenuation in the left dorsolateral prefrontal cortex response to a satiating meal using gyral regions-of-interest. J Am Coll Nutr 2009; 28: $667-673$.

14 Zanto TP, Rubens MT, Thangavel A, Gazzaley A. Causal role of the prefrontal cortex in top-down modulation of visual processing and working memory. Nat Neurosci 2011; 14: 656-661.

15 Andrews SC, Hoy KE, Enticott PG, Daskalakis ZJ, Fitzgerald PB. Improving working memory: the effect of combining cognitive activity and anodal transcranial direct current stimulation to the left dorsolateral prefrontal cortex. Brain Stimul 2011; 4: 84-89. 
16 Van den Eynde F, Claudino AM, Mogg A, Horrell L, Stahl D, Ribeiro W et al. Repetitive transcranial magnetic stimulation reduces cue-induced food craving in bulimic disorders. Biol Psychiatry 2010; 67: 793-795.

17 Brodtmann A, Puce A, Darby D, Donnan G. Regional fMRI brain activation does correlate with global brain volume. Brain Res 2009; 1259: 17-25.

18 Lind L, Fors N, Hall J, Marttala K, Stenborg A. A comparison of three different methods to evaluate endothelium-dependent vasodilation in the elderly: the Prospective Investigation of the Vasculature in Uppsala Seniors (PIVUS) study. Arterioscler Thromb Vasc Biol 2005; 25: 2368-2375.

19 Folstein MF, Folstein SE, McHugh PR. "Mini-mental state". A practical method for grading the cognitive state of patients for the clinician. J Psychiatr Res 1975; 12 $189-198$.

20 Ashburner J, Friston KJ. Voxel-based morphometry-the methods. Neuroimage 2000; 11 (6 Pt 1): $805-821$.

21 Ashburner J, Friston KJ. Unified segmentation. Neuroimage 2005; 26: 839-851.

22 Walther K, Birdsill AC, Glisky EL, Ryan L. Structural brain differences and cognitive functioning related to body mass index in older females. Hum Brain Mapp 2010 31: $1052-1064$.

23 Zakzanis KK, Mraz R, Graham SJ. An fMRI study of the Trail Making Test. Neuropsychologia 2005; 43: 1878-1886.

24 Lezak H, Loring A. Neuropsychological Assessment (4th Edition). Oxford University Press: New York, 2004.

25 Cabeza R, Nyberg L. Neural bases of learning and memory: functional neuroimaging evidence. Curr Opin Neurol 2000; 13: 415-421.

26 Whitmer RA, Gustafson DR, Barrett-Connor E, Haan MN, Gunderson EP, Yaffe K. Central obesity and increased risk of dementia more than three decades later. Neurology 2008; 71: $1057-1064$
27 Dahl A, Hassing LB, Fransson E, Berg S, Gatz M, Reynolds CA et al. Being overweight in midlife is associated with lower cognitive ability and steeper cognitive decline in late life. J Gerontol A Biol Sci Med Sci 2010; 65: 57-62.

28 Raji CA, Ho AJ, Parikshak NN, Becker JT, Lopez OL, Kuller LH et al. Brain structure and obesity. Hum Brain Mapp 2010; 31: 353-364.

29 Frings L, Mader I, Landwehrmeyer BG, Weiller C, Hull M, Huppertz HJ. Quantifying change in individual subjects affected by frontotemporal lobar degeneration using automated longitudinal MRI volumetry. Hum Brain Mapp 2011; e-pub ahead of print 26 May 2011; doi: 10.1002/hbm.21304

30 Gazdzinski S, Kornak J, Weiner MW, Meyerhoff DJ. Body mass index and magnetic resonance markers of brain integrity in adults. Ann Neurol 2008; 63: 652-657.

31 Gazdzinski S, Millin R, Kaiser LG, Durazzo TC, Mueller SG, Weiner MW et al. BMI and neuronal integrity in healthy, cognitively normal elderly: a proton magnetic resonance spectroscopy study. Obesity (Silver Spring) 2010; 18: 743-748.

32 Mendez MF, Licht EA, Shapira JS. Changes in dietary or eating behavior in frontotemporal dementia versus Alzheimer's disease. Am J Alzheimers Dis Other Demen 2008; 23: 280 - 285.

33 Volkow ND, Wang GJ, Fowler JS, Telang F. Overlapping neuronal circuits in addiction and obesity: evidence of systems pathology. Philos Trans $R$ Soc Lond $B$ Biol Sci 2008; 363: $3191-3200$.

34 Kalaria RN. Vascular basis for brain degeneration: faltering controls and risk factors for dementia. Nutr Rev 2010; 68 (Suppl 2): S74-S87.

cc This work is licensed under the Creative Commons AttributionNonCommercial-No Derivative Works 3.0 Unported License. To view a copy of this license, visit http://creativecommons.org/licenses/by-nc-nd/3.0/ 